\title{
Protocol for a systematic review of the development of depression among adolescents and young adults: psychological, biological, and contextual perspectives around the world
}

Gloria A. Pedersen', Zuzanna Zajkowska², Christian Kieling ${ }^{3}$, Kamal Gautam ${ }^{4 *}$ (D) Valeria Mondelli², Helen L. Fisher ${ }^{2}$, Johnna R. Swartz ${ }^{5}$, Abiodun Adewuya ${ }^{6}$, Rakesh Karmacharya ${ }^{7}$ and Brandon A. Kohrt ${ }^{1}$

\begin{abstract}
Background: Depression is a leading contributor to disability-adjusted life-years because of early onset and chronicity throughout the lifecycle. It is crucial to identify early predictors of depression among adolescents and young people to effectively target prevention. A gap in the literature is a comprehensive systematic review of predictors of depression among adolescents around the globe, especially in low- and middleincome countries LMICs. This review aims to identify evidence for biological, psychological, and contextual risk factors for the development of depression among adolescents and young adults (10-24 years of age) in high-income countries (HICs) and LMICs, ultimately contributing to (a) identification of potential mechanisms underlying depression development, (b) selection of common risk and protective factors as targets for detection, and (c) refinement of risk models that can be evaluated through existing cohorts in HICS and LMICs.

Methods: This review will follow the Population, Exposure, Comparison, Outcome (PI(E)CO) model and adheres to the PRISMA-P guidelines. A search strategy was developed by a multidisciplinary research consortium. Seven databases (MEDLINE via Ovid, PsycINFO, Cochrane Database of Systematic Reviews, Web of Science, Lilacs, African Journals Online, Global Health) will be searched to identify articles. Independent raters will screen and retrieve articles for inclusion, conduct quality ratings, and extract data. The Systematic Assessment of Quality in Observational Research adapted for Cultural Psychiatry Epidemiology (SAQOR-CPE) will be used to assess quality of observational studies. We will assess for publication bias using funnel plots and statistical methods. We will use narrative synthesis to present results, addressing the study's objectives following the Cochrane Handbook guidelines. Meta-analyses will be used to report summary statistics for association of risk factors with development of depression.
\end{abstract}

Discussion: This systematic review will summarize evidence-based research that examines the psychological, biological, and contextual factors contributing to the onset of depression in adolescents across the globe. Results will support the development of a model that can be evaluated in existing cohorts around the world.

\footnotetext{
* Correspondence: kgautam@tponepal.org.np

${ }^{4}$ Transcultural Psychosocial Organization Nepal (TPO Nepal), Anek Marga,

Baluwatar, G.P.O. Box 8974/CPC 612, Kathmandu, Nepal

Full list of author information is available at the end of the article
}

(c) The Author(s). 2019 Open Access This article is distributed under the terms of the Creative Commons Attribution 4.0 International License (http://creativecommons.org/licenses/by/4.0/), which permits unrestricted use, distribution, and reproduction in any medium, provided you give appropriate credit to the original author(s) and the source, provide a link to the Creative Commons license, and indicate if changes were made. The Creative Commons Public Domain Dedication waiver (http://creativecommons.org/publicdomain/zero/1.0/) applies to the data made available in this article, unless otherwise stated. 
(Continued from previous page)

Systematic review registration: PROSPERO registration CRD42018103973.

Keywords: Adolescent, Young adult, Depression, Risk factors, Protective factors, Early diagnosis, Developing countries, Neurosciences, Review, Risk assessment,

\section{Background}

The onset for most mental illnesses is during adolescence and early adulthood [1, 2]. This burden of adolescent mental illness is disproportionately borne by young people in low- and middle-income countries (LMICs) who comprise nearly $90 \%$ of the world's youth and who have the least access to services [3-6]. Moreover, one out of three suicides worldwide occurs among adolescents in LMICs [7]. Among mental illnesses, depression is the leading contributor to disability-adjusted life years-in part because of early-onset and chronicity throughout the lifecycle [8]. Therefore, there is a crucial public health need to identify early predictors of depression among adolescents and young people, with special attention to LMICs given the greatest burden and least research in these settings [4]. One key gap in the literature is a systematic review of the predictors of depression among adolescents and young people focusing on the global literature. Therefore, this systematic review will focus on early-life risk and protective factors for depression including studies from both high-income countries (HICs) and LMICs.

Previous reviews have been conducted on risk factors for adolescent depression, although currently, the reviews are focused on specific risk factors and overall generalizability of the evidence is limited, with most reviews only able to report on HICs. For example, Kohler and colleagues conducted an umbrella review mapping risk factors for depression over the lifespan, but with a focus on only environmental factors [9]. Cairns et al. [10] conducted a systematic review on risk and protective factors for adolescent depression, but, focusing only on modifiable factors, the review was not able to address contextual factors such as those at the community or family level. In the same review, of the 113 included studies, $97 \%$ of them reported on populations from HICs, a trend found in similar reviews [11, 12]. Moreover, when considering a global perspective on the development of depression, it is important to consider a wider range for the age group of interest (e.g., youth under the age of 25 years old) to account for variance in contextual factors that may play a role in the development of the adolescent brain [13, 14], particularly in LMICs. Currently, of the 12 adolescent systematic reviews found relevant to this review's topic, only 1 included ages 25 and under [15]; all others included ages 19 years or younger [10-12, 16-24].

Upon completion of this systematic review, we hope to address the above gaps in the literature, as well as contribute to the following endeavors:

- Identify common risk and protective factors across global studies as potential mechanisms underlying the development of depression in adolescence;

- Identify common risk factors as potential targets for early detection and interventions;

- Identify regional and other potential populationspecific variation in risk pathways for depression among adolescents as contextual modifiers for the focus of research and intervention adaptation;

- Identify common risk and protective factors to refine predictive models that can be evaluated using existing cohorts in HICs as well as in LMICs.

\section{Objectives}

The primary objective of this review is to identify the evidence for biological, psychological, and contextual risk factors for the development of depression among adolescents (25 years and younger) in HICs and LMICs. The secondary objectives are to identify the evidence for risk and protective factors specifically found within prospective longitudinal observational studies in HICs and LMICs, to identify the evidence for risk and protective factors specifically found within cross-sectional studies conducted in LMICs, and to identify the evidence for a relationship of biological and contextual risk factors with depression in adolescents in HICs and LMICs.

\section{Methods}

This protocol was developed in accordance with the Preferred Reporting Items for Systematic Review and Meta-Analysis Protocols (PRISMA-P) 2015 checklist [25]. This protocol has been registered in the International Prospective Register of Systematic Reviews (PROSPERO), study protocol registration CRD42018103973. 


\section{Operationalization of key constructs \\ Adolescence}

The World Health Organization (WHO) recognizes persons aged 10-19 as adolescents and those aged 15-24 as youth, with a comprehensive category of young people referring to ages 10-24 years [13]. Persons between the ages of 10-24 may be defined as adolescents as it is noted that youth changes with circumstances, especially when considering changes in settings demographically, financially, economically, and socio-culturally [14]. Therefore, for the purposes of this review, we will follow the WHO categorization of young people as 10-24 years of age, which is inclusive of adolescence [26].

\section{Biomarker}

We operationalize biomarker as "a biological characteristic objectively measured and evaluated for indications of normal biological or pathological processes, or a response to a therapeutic intervention" [27].

\section{Depression}

Usually defined as a common mental disorder, depression is characterized by persistent sadness (or irritability in the case of adolescents) and a loss of interest in activities, accompanied by an inability to carry out daily activities. Symptoms range from mild to moderate and may include (but are not limited to) several of the following: loss of energy, change in appetite, anxiety, restlessness, feelings of worthlessness, guilt, hopelessness, indecisiveness, sleeping more or less, or thoughts of self-harm or suicide. Depression is globally experienced on a continuum $[28,29]$ and for the purposes of this review will be defined by clinical diagnoses, structured clinical interviews, self-report rating scales, or medical records. The supplemental file includes a list of diagnoses and associated codes meeting the criteria for inclusion for the Diagnostic and Statistical Manual of Mental Disorders and International Classification of Disease (see Additional file 1).

\section{Low- and middle-income countries}

Low-income economies are $\$ 1025$ or less gross national income (GNI) per capita in 2015; lower middle-income economies are those with a GNI per capita between $\$ 1026$ and \$4035; upper middle-income economies are those with a GNI per capita between \$4036 and \$12,475; and high-income economies are those with a GNI per capita of $\$ 12$, 476 or more (https://blogs.worldbank.org/opendata/ new-country-classifications-2016). For countries that have changed economic status during the period of longitudinal data collection, we will require that the country met the World Bank criteria for an LMIC lending category at the time of initiating the cohort.

\section{Mechanisms of action/mechanisms of change}

This review will use the National Institute of Mental Health (NIMH) definition of mechanism of action as a "target of engagement" which may create the potential for development ("go" outcome) or reject this potential ("no-go" outcome) and its ability to modify disease, behavior, or functional outcomes; in other words, it is the basis for the effect and may also be the process or steps responsible for a therapeutic outcome [30-32].

\section{Mediators and moderators}

A mediator is operationalized as an intervening variable which lies on the causal pathway between the exposure and outcome; it may provide additional information as to how a change or outcome came about, but it is not necessarily a mechanism for change [30,33]. A moderator is operationalized as a characteristic that influences the direction or magnitude of the relationship between an independent and dependent variable [30].

\section{Protective and risk factors}

Using Cairns and colleagues' definitions (p. 63), we will operationalize a protective factor as "an antecedent condition associated with a decrease in the likelihood of the outcome of interest" [10,34] and a risk factor as "an antecedent condition associated with an increase in the likelihood of the outcome of interest" [10, 34].

\section{Study eligibility criteria \\ Types of studies}

For the initial search of risk and protective factors for depression in adolescence in HICs and LMICs, studies using longitudinal prospective designs will be identified. A second search will focus specifically on LMICs to examine cross-sectional study designs due to the lack of longitudinal studies evidenced in these settings. Finally, when considering the relationship between biological, psychological, and contextual factors and adolescent depression, we will include longitudinal and cross-sectional studies and intervention and prevention trials in order to capture any biological or contextual factors that may be evidenced while considering the gap in longitudinal study designs conducted in LMICs. Trials or experimental designs will only be included when examining biological factors in order to expand the pool of potential studies. Discussion papers, letters, editorials, case 
studies or case series, and qualitative studies without a quantitative element will be excluded.

\section{Type of participants and settings}

Both sexes in adolescent populations will be included in this review. Included studies will require a depression outcome (as defined by a categorical DSM/ICD diagnosis, clinical diagnosis, medical records, structured interview, or self-report measure). Eligible populations will be young people in high-, middle-, and low-income countries who are under the age of 25 years at study baseline and have been evaluated for depression with at least one subsequent time point of at least 6 months. The LMIC-specific analysis will also include young people living in LMICs who are under the age of 25 years who have been evaluated for the presence of depression or depressive symptoms. The biological factors' objective will include adolescents in high-, middle-, and low-income countries who are under the age of 25 years and were evaluated for depression and for biological markers of interest, as well as psychological or contextual markers associated with depression. Given the high rates of psychiatric comorbidity, populations with depression plus other psychiatric conditions (including substance abuse) will be included for the risk and protecte factors part of the review. Studies limited only to specific medical subpopulations (e.g., only young people with HIV, diabetes, intellectual disabilities) that do not include comparative populations will be excluded. Any longitudinal studies with an initial prospective time point of under 6 months will also be excluded. Medication or other treatment status will not be an exclusion criterion, but information will be reported on these whenever available.

\section{Type of exposures}

The following factors will be evaluated for association with depression among young people:

- Contextual factors associated with an increased risk for developing major depression during adolescence (this review will include demographic, economic, family, neighborhood, environmental, and social and cultural factors; in addition, we will explore life events and traumatic exposures);

- Biological markers associated with an increased risk for developing major depression during adolescence. This review will focus on studies that address the following: hypothalamicpituitary-adrenal axis (HPA axis), inflammation, endocannabinoids, vitamins, polyunsaturated fatty acids (PUFAs), hormones, neurotrophic factor, neurotransmitters, telomere/gene length, neuroplasticity, and gene expression (including mRNA quantification);

- Brain-related abnormalities in individuals at risk for major depression during adolescence (this review will focus on magnetic resonance imaging studies, both structural and functional, assessing children/adolescents at risk);

- Psychological factors associated with increased or decreased risk for developing major depression during adolescence (this review will include factors such as self-esteem, locus of control, cognitive biases, emotional regulation, reward responsivity, and learned helplessness).

\section{Comparators}

Comparison groups will vary between objectives (see Appendix 1) but will broadly include adolescents who are not exposed to the factor of interest prior to the time of assessment or adolescents who do not develop depression during the assessed periods of adolescence and young adult development.

\section{Outcomes}

The primary outcome will be development of depression among young people with a cutoff of 25 years of age or younger and also allowing for studies that include earlier endpoints of depression development, in any population, i.e., high-, middle-, and low-income countries. The primary outcome measures of depression may include self-reports, adultinformant reports, structured observations in clinical settings, and clinical records.

\section{Timing of outcome assessment}

Eligible studies should include assessment of depression during at least one time period between the ages of 10 and 24 years and a follow-up time point at least 6 months later.

\section{Search strategy for identifying relevant studies}

We will search the following electronic databases to identify potentially relevant studies for our review: MEDLINE (via Ovid), PsycINFO, Cochrane Database of Systematic Reviews, Web of Science (Core Collection), Lilacs, African Journals Online, and Global Health. In addition to these electronic databases, we also plan to identify relevant studies by reviewing reference lists of eligible studies and review articles and by contacting experts and authors of eligible studies by email. Relevant systematic reviews from the database outputs will be hand-searched for any eligible studies which may have been missed. Only 
published research in academic journals will be used for objectives 1 and 3 of this review (see below). Searches will be conducted in English, though publication language will not be a limiter. The Boolean operator "OR" will be used to find articles with one or more search terms and synonyms and "AND" to combine the different concepts for relevant articles. Where applicable, MeSH subheadings will be used, and "keywords" where relevant for given databases. For objective 2 (see below), which will use data from LMIC, we will expand our search to grey literature. Some studies of adolescents in LMIC are presented in reports of nongovernmental organizations, and these findings may not be reported in the academic literature. Therefore, we will review reports from international and national nongovernmental organizations and will attempt to identify additional literature through listservs used by mental health psychosocial practitioners working in nongovernmental organizations.

We will use truncation and wildcards to account for UK and US spelling and terminology and abbreviation. The concept combinations will vary with objective (see Appendix 1), but the three main concepts combined will include Population AND Exposure AND Outcome, for example, adolescence AND risk/protective factors AND depression. Years of publication will not be used to limit the search output, but extraction will begin in descending chronological order, assessed at the first 10 years for sufficient robust evidence, then proceed to the next 10 years or more if deemed necessary to capture representative data.

Searches will be re-run before the date of final analyses to identify recent publications in the field.

Objective 1-identify risk and protective factors associated with the development of depression among adolescents and young people in populations evaluated for at least 6 months (i.e., longitudinal samples).

Sample MEDLINE (Ovid) search:

Adolescent/ OR "adolesc" AND Depression/ OR Depressive disorder/ AND Longitudinal Studies/ OR "longitudinal"

Objective 2-identify risk and protective factors associated with depression among adolescents and young people in LMICs.

Sample MEDLINE (Ovid) search:

Adolescent/ OR "adolesc"*" AND Depression/ OR Depressive disorder/ AND Developing Countries/ OR "developing countr"” OR World Bank List (as keywords)

Objective 3-identify risk and protective factors associated with biological markers, brain related abnormalities, and context among adolescents and young people.
Sample MEDLINE (Ovid) search:

Adolescent/ OR "adolesc" AND Depression/ OR Depressive disorder/ AND "HPA axis" OR Pituitary-Adrenal System

See Appendix 1 for full MEDLINE search, Objective 1.

\section{Data collection \\ Selection of studies}

A charting form will be used to identify studies to be included in the review according to the inclusion and exclusion criteria. Two review authors will independently review titles and abstracts of studies identified through the search strategy and those from additional resources (e.g., contacting authors and searching reference lists of included papers, which will be done through hand searches, Web of Science citation records, or Scopus when appropriate) to determine whether studies potentially meet the inclusion criteria. To assess for study eligibility, two reviewers will independently review the full publication texts of studies identified through title and abstract review. They will record justification for exclusion for each excluded study. A third author will resolve disagreements between the two reviewers.

\section{Retrieval of studies}

The full text of the selected articles will be retrieved. When a full article is not available through the database or university library services, it will be searched through ResearchGate and Google Scholar, or a request to share the full article will be sent by email to the corresponding author. After the request has been sent, a reminder will be sent after a week. If there is no response within 2 weeks from the reminder, this document will be excluded from the systematic review.

\section{Data extraction and management/coding}

Extraction fields will include, but not be limited to, article information (publication year, journal), study characteristics (country, urban vs. rural setting, funding), population characteristics (age at enrolment, current age, number of longitudinal assessments), depression assessment (depression criteria, duration of depression, assessment tool, co-morbidity), risk factors (poverty, trauma, family history), protective factors (education, social capital), assessment tool (instrument names and psychometric properties for depression, risk, and protective factors), and biological variables (interleukins, cortisol, galvanic skin response, neuroimaging). Two authors will independently extract data from the published reports of included studies using a standardized, electronic form (e.g., in Microsoft Excel), which will be developed and pre-piloted to assure interrater agreement. We will attempt to collect 
missing information by contacting the corresponding authors via email.

\section{Assessment of risk of bias in included studies}

Two reviewers will independently assess the risk of bias in included studies by evaluating the following domains:

- Recruitment and sampling strategy

- Follow-up strategy, retention, and selective attrition

- Completeness of outcome data

- Validity and other psychometrics of depression outcomes and other tools used

- Selective outcome reporting

- Other sources of bias related to context, procedures, and reporting

We will assess the quality of observational studies using the Systematic Assessment of Quality in Observational Research (SAQOR) [35] and the adapted SAQOR Cultural Psychiatry Epidemiology (SAQOR-CPE) [36]. If any intervention studies are included, we will limit the analyses to be pre-treatment findings or control groups. We will apply the SAQOR-CPE to the samples of interest in the intervention studies. Quality level will be recorded as high, moderate, low, or very low risk of bias for each domain, and bias rating will be compared between the two author reviewers. A third author will resolve discrepancies. Emails to authors will be used to obtain additional information to reduce sources of bias and adjust risk.

\section{Data analysis}

\section{Measurement of exposure effect}

The primary comparison for this systematic review will be development of depression before the age of 25 years old. We anticipate that there will be substantial heterogeneity between studies (including reporting differences) that may limit our ability to conduct quantitative syntheses of findings. Similarly, we anticipate heterogeneity in measurement of depression and depressive symptoms, as well as validation, evaluation, and translation of tools. Where there is adequate homogeneity, we will synthesize results using random-effects meta-analysis. We will use standardized mean differences (SMDs) for continuous outcomes and risk ratios for binary outcomes and calculate $95 \%$ confidence intervals and two-sided $p$ values for each outcome. When applicable, we will use SMDs to generate pooled estimates to compare different types of risk factors. We will conduct all quantitative tests using appropriate techniques in Stata, R, and in Comprehensive Meta-Analysis (Biostat, Englewood, NJ, https:// www.meta-analysis.com/). We will follow Ioannidis and colleagues [37] recommendations for conducting metaanalyses and reporting summary statistics. Rather than a priori excluding use of meta-analysis or setting specific criteria for degree of heterogeneity, Ioannidis and colleagues state that it is preferable to present the summary statistic, while clearly documenting the heterogeneity and its possible causes. We employ methods recommended by Ioannidis and colleagues to report and adjust for heterogeneity.

\section{Unit of analysis issues}

The study population will be the unit of analysis. We do not anticipate obtaining sufficient access to individuallevel data to conduct individual-level analyses. Studies that only include cluster-level analysis will not be included.

\section{Dealing with missing data}

For missing measures of precision (confidence intervals or standard deviations) that remain unavailable after attempting to contact study authors, we will assume the highest variability observed within the group of studies being analyzed.

\section{Assessment of reporting bias}

We will assess for publication bias using funnel plots. In addition, we will use the approach outlined by Begg and Mazumdar [38] to statistically test for publication bias.

\section{Data synthesis}

We will provide a narrative synthesis of findings from the included studies. Following Cochrane guidelines [39], we will provide narrative synthesis information separately by study type. From the studies, we develop a conceptual framework to identify themes. This will be done at the full-text stage after reviewing the manuscripts. We will provide descriptives, compare studies on characteristics, tabulate results to identify patterns across studies, use vote counting, and translate data for thematic and content analyses per Cochrane recommendations. For quantitative synthesis, we will provide summaries of the primary outcome measures for each study. We will include forest plots summarizing results of individual studies and the meta-analyses. Summaries of depression outcomes will be subcategorized accordingly by self-report, parent or teacher report, clinical instrument, or healthcare service diagnosis. Also, specifically for the biological factors, summaries will be stratified by those with and without substance abuse diagnoses. We will run meta-analyses for each objective with meta-regression where appropriate, as well as assess methodological issues and conduct sensitivity analyses.

\section{Subgroup analysis and assessment of heterogeneity}

If possible, we will conduct subgroup analyses based on HICs vs. LMICs, by age of onset, co-morbidities, outcome 
type (e.g., self-report, clinical interview, medical records), geographic region, and population settings (primary care, non-psychiatric specialty services, communities, religious centers, schools). Some included studies may report onset of depression after 25 years of age for some participants. Therefore, we will potentially conduct subgroup analyses by age group of onset: those who have developed depression before the age of 25 and those who have developed it after the age of 25 for studies that follow participants into adulthood. Subgroup analyses will be conducted in Comprehensive Meta-Analysis, including analysis of statistical difference among subgroups. We will assess heterogeneity using the chi-squared test, $I$-squared statistic, and prediction interval [40]. We will evaluate for potential sources of heterogeneity. We will address heterogeneity by using random-effects meta-analysis models and sensitivity analyses. We will use the approach of referring to "low, moderate, and high" heterogeneity at the $I^{2}$ thresholds of $25 \%, 50 \%$, and $75 \%$, with the caveat that quantification of heterogeneity will be considered only one marker of variability across studies [41] and the concerns raised by Cuijpers [42] that even point estimates of $I^{2}$ may have wide confidence intervals extending into the ranges of high heterogeneity.

\section{Sensitivity analysis}

We will conduct sensitivity analyses as recommended by the Cochrane Handbook (Section 9.7) [43]. For example, sensitivity analyses will be conducted removing the studies with the strongest effects sizes and studies with the largest sample sizes. We will follow the approach described in Patsopoulos et al. [44] of excluding up to two studies to see if heterogeneity crosses the threshold below $50 \%$ or $25 \%$.

\section{Assessing overall confidence in cumulative evidence}

We will use the Confidence in the Evidence from $\mathrm{Re}$ views of Qualitative research (CERQual) tool to assess confidence in the qualitative syntheses. Similarly, we will use SAQOR-CPE in reference to quantitative reviews.

\section{Presentation of results}

We will present the results of our systematic review through a narrative description addressing the study's objectives and through charts and tables as recommended in the Cochrane Handbook (Section 11) [43]. First, we will tabulate data from our search process into a flowchart indicating the number of studies identified and excluded at key steps, according to the PRISMA guidelines [25]. Second, we will summarize information on individual studies in a "Characteristics of Included Studies" table. Third, we will present "Data and Analyses" tables and forest plots to present outcome data from individual studies and from any meta-analyses. Fourth, we will present a "Summary of Findings" table to summarize key data available, magnitude of effect of the risk and protective factors studied, and quality of evidence addressing the key study objectives.

\section{Discussion}

There is a crucial public health need to identify early predictors of depression among adolescents and young people around the globe, with special attention to LMICs given that the greatest burden and least research is found in these settings. This systematic review aims to address gaps in the review literature and will summarize evidence-based research which looks at the psychological, biological, and contextual factors contributing to the onset of depression in adolescents and young people across the globe. Recommendations will be made to inform future research priorities. The results will support the exploration of the feasibility and acceptability of early detection and interventions in LMICs and support the development of a common risk factor assessment model that can be evaluated using existing cohorts in HICs as well as in LMICs. These results will inform relevant academic and practitioner communities, key stakeholders such as policy- and decision-makers, and adolescents and their families.

\section{Appendix 1}

Table 1 MEDLINE (Ovid) search, objective 1

1. ADOLESCENT/

2. (adolesc* or "youth" or "teen" or "young people" or "young person*").mp. [mp=title, abstract, original title, name of substance word, subject heading word, floating sub-heading word, keyword heading word, protocol supplementary concept word, rare disease supplementary concept word, unique identifier, synonyms]

3. Young Adult/

4. 1 or 2 or 3

5. DEPRESSION/

6. Depressive Disorder/

7. Depressive Disorder, Major/

8. ("depressive disorder" or "symptoms of depression" or "major depressive disorder").mp. [mp=title, abstract, original title, name of substance word, subject heading word, floating sub-heading word, keyword heading word, protocol supplementary concept word, rare disease supplementary concept word, unique identifier, synonyms]

9. 5 or 6 or 7 or 8

10. LONGITUDINAL STUDIES/

11. Prospective Studies/

12. Observational Study/

13. (longitudinal or prospective or "observational stud").mp. [mp=title, abstract, original title, name of substance word, subject heading word, floating sub-heading word, keyword heading word, protocol supplementary concept word, rare disease supplementary concept word, unique identifier, synonyms]

14. 10 or 11 or 12 or 13

15. 4 and 9 and 14 


\section{Additional file}

Additional file 1: List of diagnoses and associated codes meeting the criteria for inclusion for the Diagnostic and Statistical Manual of Mental Disorders and International Classification of Disease. (DOCX 12 kb)

\section{Abbreviations}

DSM: Diagnostic and Statistical Manual of Mental Disorders; GNI: Gross national income; HIC: High-income country; HPA axis: Hypothalamic-pituitaryadrenal axis; ICD: International Classification of Diseases; LMIC: Low- and middle-income countries; NIMH: National Institutes of Mental Health; PI(E)COS: Population, Exposure, Comparators, Outcomes, and Study designs; PRISMA: Preferred Reporting Items for Systematic Reviews and MetaAnalyses; PRISMA-P: Preferred Reporting Items for Systematic Review and Meta-analysis Protocols; PROSPERO: International Prospective Register of Systematic Reviews; PUFAs: Polyunsaturated fatty acids; RNA: Ribonucleic acid; SAQOR: Systematic Assessment of Quality in Observational Research SAQOR-CPE: Systematic Assessment of Quality in Observational Research-Cultural Psychiatry Epidemiology; SMD: Standardized mean differences; WHO: World Health Organization

\section{Acknowledgements}

We would like to thank Tomas Alvarez, Jyoti Bohara, and Eryn Nagel for their support in preliminary screening and initial title and abstract screening of articles. We thank Pedro Manfro and Thiago Botter-Maio Rocha for their assistance with manuscript revisions.

\section{Authors' contributions}

BAK and CK conceptualized the review. GAP and BAK drafted the protocol and manuscript. All authors critically appraised and edited the manuscript. GAP created the inclusion and exclusion criteria and definitions, which were reviewed and revised by co-authors. GAP and $\mathrm{ZZ}$ created the search terms and conducted preliminary searches. GAP completed the PROSPERO registration. All authors contributed to the revision and finalization of the manuscript. All authors read and approved the final manuscript.

\section{Funding}

MQ Transforming Mental Health Charity, Brighter Futures grant named "Identification of Depression Early in Adolescence", Grant \#MQBF/1 IDEA. Additional support was provided by the UK Medical Research Council (MC_PC_MR/R019460/1) and the Academy of Medical Sciences (GCRFNG\100281).

CK has received support from Brazilian governmental research funding agencies (Conselho Nacional de Desenvolvimento Científico e Tecnológico [CNPq], Coordenação de Aperfeiçoamento de Pessoal de Nível Superior [CAPES], and Fundação de Amparo à Pesquisa do Estado do Rio Grande do Sul [Fapergs]).

VM has been supported by the National Institute for Health Research (NIHR) Mental Health Biomedical Research Centre at South London and Maudsley NHS Foundation Trust and King's College London. The views expressed are those of the authors and not necessarily those of the National Health Service, the NIHR, or the Department of Health. BAK is supported by the US National Institute of Mental Health (K01MH104310, R21MH111280).

\section{Availability of data and materials} Not applicable.

\section{Ethics approval and consent to participate}

Not applicable.

\section{Consent for publication}

Not applicable.

\section{Competing interests}

VM has received research funding from Johnson \& Johnson, a pharmaceutical company interested in the development of anti-inflammatory strategies for depression, but the research described in this paper is unrelated to this funding. All other authors declare that they have no competing interests.

\section{Author details}

${ }^{1}$ School of Medicine and Health Sciences, Division of Global Mental Health, 2120 L St NW, Ste 600, Washington DC 20037, USA. Institute of Psychiatry, Psychology \& Neuroscience, King's College London, 16 De Crespigny Park, London SE5 8AF, UK. ${ }^{3}$ Department of Psychiatry and Child \& Adolescent Psychiatry Division, Universidade Federal do Rio Grande do Sul, Hospital de Clínicas de Porto Alegre, Rua Ramiro Barcelos, 2350 - 400N, Porto Alegre, RS 90035-903, Brazil. ${ }^{4}$ Transcultural Psychosocial Organization Nepal (TPO Nepal), Anek Marga, Baluwatar, G.P.O. Box 8974/CPC 612, Kathmandu, Nepal. ${ }^{5}$ Department of Human Ecology, University of California Davis, One Shields Ave, Davis, CA 95616, USA. 'Department of Behavioral Medicine, Lagos State University College of Medicine, 1-5 Oba Akinjobi Way, G.R.A., Ikeja P.M.B, Lagos 21266, Nigeria. ${ }^{7}$ Center for Human Genetic Research, Massachusetts General Hospital, 185 Cambridge Street, Boston, MA 02114, USA.

Received: 10 October 2018 Accepted: 12 July 2019

Published online: 20 July 2019

\section{References}

1. Kessler RC, Amminger GP, Aguilar-Gaxiola S, Alonso J, Lee S, Ustun TB. Age of onset of mental disorders: a review of recent literature. Curr Opin Psychiatr. 2007:20(4):359-64.

2. Kim-Cohen J, Caspi A, Moffitt TE, Harrington H, Milne BJ, Poulton R. Prior juvenile diagnoses in adults with mental disorder. Arch Gen Psychiatry. 2003;60:709-17.

3. Erskine HE, Moffitt TE, Copeland WE, Costello EJ, Ferrari AJ, Patton G, et al. A heavy burden on young minds: the global burden of mental and substance use disorders in children and youth. Psychol Med. 2015:45(07):1551-63.

4. Kieling C, Baker-Henningham H, Belfer M, Conti G, Ertem I, Omigbodun O, et al. Child and adolescent mental health worldwide: evidence for action. Lancet. 2011;378(9801):1515-25.

5. Leckman JF, Leventhal BL. Editorial: a global perspective on child and adolescent mental health. J Child Psychol Psychiatry Allied Discip. 2008:49(3):221-5

6. Belfer ML. Child and adolescent mental disorders: the magnitude of the problem across the globe. J Child Psychol Psychiatry Allied Discip. 2008; 49(3):226-36.

7. WHO. Preventing suicide: a global imperative. Geneva: World Health Organization; 2014

8. Whiteford HA, Degenhardt L, Rehm J, Baxter AJ, Ferrari AJ, Erskine HE, et al. Global burden of disease attributable to mental and substance use disorders: findings from the Global Burden of Disease Study 2010. Lancet. 2016:382(9904):1575-86.

9. Kohler CA, Evangelou E, Stubbs B, Solmi M, Veronese N, Belbasis L, et al. Mapping risk factors for depression across the lifespan: an umbrella review of evidence from meta-analyses and Mendelian randomization studies. J Psychiatr Res. 2018;103:189-207.

10. Cairns KE, Yap MB, Pilkington PD, Jorm AF. Risk and protective factors for depression that adolescents can modify: a systematic review and meta-analysis of longitudinal studies. J Affect Disord. 2014;169:61-75.

11. Reiss F. Socioeconomic inequalities and mental health problems in children and adolescents: a systematic review. Soc Sci Med. 2013;90: 24-31.

12. Bor W, Dean AJ, Najman J, Hayatbakhsh R. Are child and adolescent mental health problems increasing in the 21 st century? A systematic review. Aust N Z J Psychiatry. 2014;48(7):606-16.

13. WHO. Youth and health risks. 2011. Contract No.: A64/25

14. United Nations Department of Economic and Social Affairs. Definition of youth. UNDESA, United Nations Department of Economic and Social Affairs; 2010. http://undesadspd.org/Youth.aspx.

15. Kerestes R, Davey CG, Stephanou K, Whittle S, Harrison BJ. Functional brain imaging studies of youth depression: a systematic review. Neuroimage Clin. 2014;4:209-31.

16. Stirling K, Toumbourou JW, Rowland B. Community factors influencing child and adolescent depression: a systematic review and meta-analysis. Aust N Z J Psychiatry. 2015:49(10):869-86.

17. Sanger C, lles JE, Andrew CS, Ramchandani PG. Associations between postnatal maternal depression and psychological outcomes in adolescent offspring: a systematic review. Arch Womens Ment Health. 2015;18(2):147-62. 
18. Yap MB, Pilkington PD, Ryan SM, Jorm AF. Parental factors associated with depression and anxiety in young people: a systematic review and meta-analysis. J Affect Disord. 2014;156:8-23.

19. Dardas LA, Silva SG, Smoski MJ, Noonan D, Simmons LA. The prevalence of depressive symptoms among Arab adolescents: findings from Jordan. Public Health Nurs. 2018;35(2):100-8.

20. Polanczyk GV, Salum GA, Sugaya LS, Caye A, Rohde LA. Annual research review: a meta-analysis of the worldwide prevalence of mental disorders in children and adolescents. J Child Psychol Psychiatry Allied Discip. 2015;56(3):345-65.

21. American Psychological Association. The dexamethasone suppression test: an overview of its current status in psychiatry. The APA Task Force on laboratory tests in psychiatry. Am J Psychiatry. 1987;144(10):1253-62.

22. Koenig J, Kemp AH, Beauchaine TP, Thayer JF, Kaess M. Depression and resting state heart rate variability in children and adolescents - a systematic review and meta-analysis. Clin Psychol Rev. 2016;46:136-50.

23. Braithwaite EC, O'Connor RM, Degli-Esposti M, Luke N, Bowes L. Modifiable predictors of depression following childhood maltreatment: a systematic review and meta-analysis. Transl Psychiatry. 2017;7(7):e1162.

24. Vilgis V, Silk TJ, Vance A. Executive function and attention in children and adolescents with depressive disorders: a systematic review. Eur Child Adolesc Psychiatry. 2015;24(4):365-84.

25. Moher D, Shamseer L, Clarke M, Ghersi D, Liberati A, Petticrew M, et al. Preferred reporting items for systematic review and meta-analysis protocols (PRISMA-P) 2015 statement. Syst Rev. 2015;4(1):1.

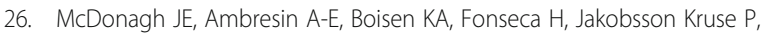
Meynard A, et al. The age of adolescence ... and young adulthood. Lancet Child Adolesc Health. 2018;2(4):e6.

27. Nature. Biomarkers: Macmillan Publishers Limited; 2018 [Available from: https://www.nature.com/subjects/biomarkers.

28. World Health Organization. Depression: What you should know. In: Organization WH, editor. World Health Organization; 2017. https://www. who.int/campaigns/world-healthday/2017/handouts-depression/what-youshould-know/en/.

29. Patel V. Talking sensibly about depression. PLoS Med. 2017;14(4): e1002257.

30. Kazdin AE. Mediators and mechanisms of change in psychotherapy research. Annu Rev Clin Psychol. 2007;3(1):1-27.

31. Bosqui TJ, Marshoud B. Mechanisms of change for interventions aimed at improving the wellbeing, mental health and resilience of children and adolescents affected by war and armed conflict: a systematic review of reviews. Confl Health. 2018;12:15.

32. Insel TR, Gogtay N. National institute of mental health clinical trials: new opportunities, new expectations. JAMA Psychiatry. 2014;71(7):745-6.

33. Stewart R. Inference 1: chance, bias, and confounding. In: Prince M, Stewart R, Ford T, Hotopf M, editors. Practical psychiatric epidemiology. Oxford: Oxford University Press; 2003.

34. Kazdin AE, Kraemer HC, Kessler RC, Kupfer DJ, Offord DR. Contributions of risk-factor research to developmental psychopathology. Clin Psychol Rev. 1997;17(4):375-406.

35. Ross LE, Grigoriadis S, Mamisashvili L, Koren G, Steiner M, Dennis CL, et al. Quality assessment of observational studies in psychiatry: an example from perinatal psychiatric research. Int J Methods Psychiatr Res. 2011;20(4):224-34.

36. Kohrt BA, Rasmussen A, Kaiser BN, Haroz EE, Maharjan SM, Mutamba BB, et al. Cultural concepts of distress and psychiatric disorders: literature review and research recommendations for global mental health epidemiology. Int J Epidemiol. 2014;43(2):365-406.

37. loannidis JPA, Patsopoulos NA, Rothstein HR. Reasons or excuses for avoiding meta-analysis in forest plots. BMJ. 2008;336(7658):1413.

38. Begg CB, Mazumdar M. Operating characteristics of a rank correlation test for publication bias. Biometrics. 1994;50(4):1088-101.

39. Ryan R, Cochrane Consumers and Communication Review Group. Cochrane consumers and communication review group: data synthesis and analysis 2016.

40. Borenstein M, Higgins JPT, Hedges LV, Rothstein HR. Basics of metaanalysis: 12 is not an absolute measure of heterogeneity. Res Synth Methods. 2017:8(1):5-18.

41. Higgins JPT, Thompson SG, Deeks JJ, Altman DG. Measuring inconsistency in meta-analyses. BMJ. 2003;327(7414):557.
42. Cuijpers P. Meta-analyses in mental health research. A practical guide. Amsterdam: Vrije Universitat Amsterdam; 2016. Available from: https://www. researchgate.net/publication/301815425

43. Higgins J, et al. location online: https://handbook-5-1.cochrane.org/.

44. Patsopoulos NA, Evangelou E, loannidis JPA. Sensitivity of between-study heterogeneity in meta-analysis: proposed metrics and empirical evaluation. Int J Epidemiol. 2008;37(5):1148-57.

\section{Publisher's Note}

Springer Nature remains neutral with regard to jurisdictional claims in published maps and institutional affiliations.
Ready to submit your research? Choose BMC and benefit from:

- fast, convenient online submission

- thorough peer review by experienced researchers in your field

- rapid publication on acceptance

- support for research data, including large and complex data types

- gold Open Access which fosters wider collaboration and increased citations

- maximum visibility for your research: over $100 \mathrm{M}$ website views per year

At $\mathrm{BMC}$, research is always in progress.

Learn more biomedcentral.com/submissions 